\title{
Influencia del nitruro de aluminio en el crecimiento anormal de grano de aceros microaleados ${ }^{(\bullet)}$
}

\author{
J.M. Cabrera ${ }^{(*)}$, A. Al Omar ${ }^{(*)}$ y J.M. Prado ${ }^{(*)}$ \\ Resumen Tras una breve revisión de los conceptos y teorías involucradas en el control del crecimiento de \\ grano por partículas de segunda fase, se efectúa un estudio del mismo en un acero microaleado con \\ contenido medio de carbono con dos niveles de nitruro de aluminio. Se propone un sistema de \\ estudio de las distribuciones de tamaño de grano tendente a poder detectar la presencia de \\ crecimiento anormal de grano. De este modo, se construyen mapas de crecimiento anormal-tiempo- \\ temperatura que, correlacionados con las teóricas fracciones de volumen de los precipitados \\ presentes, permiten comprobar que la disolución del nitruro de aluminio es la responsable \\ fundamental de la aparición del crecimiento anormal de grano. También se muestra el efecto e \\ importancia de la velocidad de calentamiento, ya que altas velocidades pueden producir una \\ sobresaturación transitoria de precipitados que, al disolverse, pueden dar lugar a crecimiento de \\ grano anormal.
}

Palabras clave: Acero microaleado. Control del grano. Crecimiento anormal. Nitruro de aluminio.

\section{Influence of aluminium nitride on the abnormal grain growth in a microalloyed steel}

\begin{abstract}
After a brief review about concepts and theories involved in control of grain growth by second phase particles, an experimental study of a medium carbon microalloyed steel with two levels of AlN is carried out. A system to study the grain size distributions in order to detect the abnormal grain growth is proposed. Results permit to plot a map of abnormal grain growth-time-temperature. The abnormal grain growth is derived to be due to the AIN dissolution by correlating the above maps with the theoretical volume fraction of precipitates. The importance and effect of the heating rate is also shown: high heating rates can produce a transient oversaturation that can lead to abnormal growing during the dissolution.
\end{abstract}

Keywords: Microalloyed steel. Grain control. Abnormal growing. Aluminium nitride.

\section{INTRODUCCIÓN}

La aplicación de los aceros microaleados a la forja en caliente es un tema en creciente estudio, debido al ahorro que representa su utilización con respecto a los aceros convencionales de temple y revenido (1-3). Este ahorro se debe a la supresión

(•) Trabajo recibido el día 18 de abril de 1994.

(*) Dpto. de Ciencia de Materiales e Ingeniería Metalúrgica. Universidad Politécnica de Cataluña. Avda. Diagonal, 647. 08028-Barcelona (España). del tratamiento térmico subsiguiente al conformado, puesto que los mecanismos de obtención de propiedades mecánicas en los aceros microaleados son los de endurecimiento por precipitación y afino de grano. Las propiedades mecánicas de los aceros microaleados se obtienen, por lo tanto, directamente después del conformado en caliente y, para composiciones idénticas, las citadas propiedades se ven influidas por las temperaturas de calentamiento, por las temperaturas y condiciones de conformado (deformación y velocidad de deformación) y por la velocidad de enfriamiento. 
Para obtener una buena combinación de tenacidad y resistencia, condición de aplicación industrial de los aceros microaleados, se requiere una estructura de grano fino junto a una apropiada estructura ferrítico-perlítica, lo cual puede determinarse mediante:

- El tamaño de grano austenítico a la temperatura de calentamiento.

- Las condiciones de conformado en caliente y posterior enfriamiento.

El presente estudio se centrará en el primer punto.

Una de las formas más importantes de controlar el tamaño de grano en una aleación es por medio de la adición de una dispersión de partículas de segunda fase. Éste es uno de los papeles reservados a los elementos microaleantes de los aceros objeto de este estudio.

Usualmente, la presencia de partículas de segunda fase lleva aparejada una serie de fenómenos (4), como son:

- Control del grano: Tiene lugar cuando el material se calienta por debajo de la temperatura de disolución de los precipitados. El grano crece hasta un valor máximo a partir del cual se mantiene constante, controlado. Este tamaño de grano máximo aumenta con la temperatura de calentamiento y/o con cantidades decrecientes de la fase dispersa. Los granos obtenidos en tales condiciones son de menor tamaño que los del material sin precipitados.

- Crecimiento anormal de grano: Ocurre cuando el material se calienta a temperaturas justo por debajo de la de disolución de los precipitados, donde parte de ellos están disueltos y parte coalescen. Su característica fundamental es la no constancia de parámetros topológicos, puesto que se desarrollan distribuciones no uniformes de formas y/o tamaños de grano, de manera que algunos de ellos crecen más rápidamente que el resto de la matriz en la que se engloban.

- Crecimiento normal: Tiene lugar cuando el material se calienta a temperaturas tales que la fase dispersa se disuelve totalmente o bien cuando el tamaño de grano es inferior al de control de los precipitados. Se caracteriza por el mantenimiento de una estructura de granos uniforme; la distribución de formas y/o tamaños permanece constante en todo el material mientras el tamaño de grano medio aumenta.

Debe apuntarse que la inhibición del crecimiento de grano por partículas puede ocurrir en aleaciones que contengan más de un tipo de precipitados, en cuyo caso se puede encontrar una dependencia compleja con la temperatura y el tiempo.

\section{CONTROL DEL TAMANO DE GRANO. REVISIÓN TEÓRICA}

Zener (5) fue el primero en obtener una expresión teórica que relacionaba el tamaño y fracción de volumen de las partículas precipitadas con el tamaño de grano crítico por encima del cual no se ejerce un control del mismo. Su interpretación se basa en que el crecimiento de grano cesa cuando la fuerza de anclaje ocasionada sobre el límite de grano por las partículas precipitadas se iguala con la fuerza motora que produce el crecimiento de grano.

De esta manera obtuvo la siguiente ecuación:

$$
R_{\text {crít. }}=\frac{4 r}{3 f}
$$

siendo $R_{\text {crít, }}$ el radio de grano máximo que puede controlar la fase dispersa presente, de fracción de volumen $f$ y radio medio $r$.

Las hipótesis subyacentes en la teoría de Zener son que las partículas están aleatoriamente distribuidas y tienen forma de esferas uniformes, que la energía interfacial partícula-matriz es independiente de la orientación de la matriz, que cada partícula ejerce la máxima fuerza restrictiva sobre el movimiento del límite de grano y que los granos están aislados de sus vecinos.

La teoría de Zener predice que a partir de una combinación crítica de la fracción en volumen y tamaño de los precipitados cesa el control del tamaño de grano. Generalmente, a continuación suele observarse crecimiento anormal, para más tarde volver de nuevo al crecimiento normal. La citada teoría no explica los motivos del crecimiento anormal, aunque puede definir el inicio del mismo.

Fue Hillert (6), dentro de su teoría general para el crecimiento de grano, quien dio una primera explicación de la aparición del crecimiento anormal de grano. Para ello aplicó la teoría de coalescencia de precipitados de Lifshitz y col. (7) al crecimiento de grano, asumiendo que su velocidad de crecimiento es proporcional a la diferencia de presión causada por la variación de curvatura del límite de grano, y que éstos son esféricos. De este modo, encontró que para una matriz libre de partículas de segunda fase, el tamaño de grano evoluciona con el tiempo según la siguiente ecuación:

$$
\frac{\mathrm{d} R}{\mathrm{~d} t}=\alpha M \sigma\left(\frac{1}{R_{\text {crít. }}^{\prime}}-\frac{1}{R}\right)
$$

siendo $M$ la movilidad del límite de grano; $\alpha$, una constante; $\sigma$, la energía de límite de grano por 
unidad de área; $R$, el radio de grano actual; y $R_{\text {crít.' }}^{\prime}$, el valor que anula la expresión. Si $R<R_{\text {crít. }}^{\prime}$, entonces el grano tiende a ser consumido $(\mathrm{d} R / \mathrm{d} t<0)$, mientras que si $R>R_{\text {crít. }}^{\prime}$, el grano tiende a crecer $(\mathrm{d} R / \mathrm{d} t>0)$.

Al tener en cuenta el efecto de partículas de segunda fase, Hillert añadió la fuerza de oposición calculada por Zener, de modo que obtuvo:

$$
\frac{\mathrm{d} R}{\mathrm{~d} t}=\alpha M \sigma\left(\frac{1}{R_{\text {crít. }}^{\prime}}-\frac{1}{R} \pm \frac{Z}{\alpha}\right)
$$

siendo $Z=(3 f / 4 r)$.

El signo debe escogerse de modo que se actúe contra el movimiento del límite de grano, en función de si éste crece o decrece. De la expresión anterior es fácil ver que existe un intervalo de valores de $R$ ( $R_{1}$ y $R_{2}$ en las ecuaciones [4] y [5]), entre los que la fuerza de oposición al movimiento de los límites de grano excede a la fuerza motora para el movimiento de los mismos, lo cual no es físicamente posible. En este rango debe ser $\mathrm{d} R / \mathrm{d} t=0$, es decir, los granos están anclados.

$$
\begin{aligned}
& R_{1}=\frac{R_{\text {crít. }}^{\prime}}{1+\frac{Z}{\alpha} R_{\text {crít. }}^{\prime}} \\
& R_{2}=\frac{R_{\text {crít. }}^{\prime}}{1-\frac{Z}{\alpha} R_{\text {crít. }}^{\prime}}
\end{aligned}
$$

Así, incluso en aleaciones bajo condiciones de crecimiento normal, el crecimiento está restringido a aquellos granos con radios mayores que $R_{2}$. Mediante razonamientos topológicos (al fin y al cabo los granos llenan el espacio) y el llamado modelo de defectos, Hillert demostró que $R_{\text {crít }}^{\prime}$ es proporcional al radio de grano promedio de toda la estructura. En consecuencia, $R_{2}$ aumenta con el crecimiento normal y cada vez pueden crecer menos granos grandes. Los efectos de esto son, por un lado, que el crecimiento de grano cesa cuando el radio medio de éste es igual al de Zener, y, por otro lado, que la distribución de tamaños se va ampliando y desarrollando una cola por la derecha. En todo el razonamiento anterior, $Z$ se mantiene constante. $\mathrm{Si}$ en condiciones de crecimiento normal inhibido varían las características de la fase dispersa (bien su fracción en volumen, bien su tamaño medio), es posible que el valor de $R_{2}$ se reduzca y sea menor que el valor máximo dentro del rango existente de tamaños de grano. En estas circunstancias, los granos de la cola derecha de la distribución de tamaños de grano anclados podrán crecer, mientras que el resto no. Esto dará lugar a un progresivo aumento de los granos más grandes (que son los únicos que pueden crecer pues el resto están anclados) y la aparición de una microestructura dúplex.

Hillert llega a establecer los rangos entre los que se observa cada tipo de crecimiento de grano. Cuando el tamaño medio es inferior a $1 /(3 Z)$, sólo puede tener lugar crecimiento normal. Si el tamaño de grano medio es superior a $1 /(2 Z)$ no puede haber crecimiento de grano. Entre ambos valores es posible la existencia de crecimiento anormal si ya existen algunos granos mucho mayores que el valor medio.

Los modelos hasta ahora comentados no predicen con exactitud el tamaño de grano de la matriz cuando hay presencia de partículas de segunda fase. Así, el modelo de Hillert predice mejor los tamaños máximos que los medios, y el modelo de Zener predice valores، del tamaño de grano medio que son aproximadamente diez veces superiores a los experimentales.

Es por ello que hasta la fecha se han propuesto diversos modelos adicionales, la mayoría de los cuales incorporan las ideas de Zener y de Hillert. Dos de los modelos más interesantes son el de Gladman y el más reciente de Ríos.

Gladman (8) añadió una serie de correcciones a la teoría de Zener, dadas las hipótesis simplificadoras de aquél. Su contribución fundamental fue la de considerar que los granos no están aislados y que cuando un grano crece, su vecino decrece. Su razonamiento le condujo a la siguiente ecuación:

$$
R_{\text {crít. }}^{\prime}=\frac{\pi r}{6 f}\left(\frac{3}{2}-\frac{2}{K}\right)
$$

donde $K$ es la relación entre los granos que crecen y el tamaño medio de los granos que se consumen, una especie de factor de heterogeneidad de la microestructura. Aún así su teoría tampoco predice con exactitud los tamaños de grano de la matriz.

Los recientes trabajos de Ríos (9) han disminuido esta discrepancia al proponer un modelo en el que se considera la energía disipada durante el movimiento de una intercara a través de una dispersión de partículas en lugar de considerar modelos de equilibrios de fuerzas como hizo Zener.

Ríos calcula la variación total del área superficial como la variación del área de los granos más la creación de la intercara grano-precipitado. El grano crecerá mientras sea posible una disminución neta de esta energía de superficie. Así, se obtiene que el crecimiento de grano cesa cuando el tamaño de 
grano medio, $R_{\text {med. }}$, es igual a un tamaño de grano crítico, $R_{\text {crít. }}$ y de valor:

$$
R_{\text {crít. }}=\frac{1}{2 S_{\mathrm{v}}^{\mathrm{p}}}
$$

siendo $S_{\mathrm{v}}^{\mathrm{p}}$ la superficie por unidad de volumen que ocupan las partículas. Para el caso de partículas esféricas de radio $r$ y fracción de volumen $f$, la expresión anterior se transforma en:

$$
R_{\text {crít. }}=\frac{r}{6 f}
$$

donde debe notarse que este $R_{\text {crít. }}$ es inferior al de Zener.

Para el análisis del crecimiento anormal, Ríos estudia el crecimiento de un grano aislado en una matriz homogénea siguiendo un planteamiento similar al de Hillert. Su análisis le lleva, al igual que a Gladman, a tener que usar un parámetro de heterogeneidad del sistema $\left(R_{\text {máx. }} / R_{\text {med. }}\right)$, de modo que define la heterogeneidad crítica $H_{\mathrm{c}}$, por encima de la cual es posible la existencia de crecimiento anormal de grano. Dicha heterogeneidad crítica tiene un valor:

$$
H_{\mathrm{c}}=\left[3-\frac{R_{\text {med. }}}{R_{\text {crít. }}}\right]^{-1}
$$

donde $R_{\text {crít. }}$ es el radio crítico de la ecuación [8].

De esta manera, Ríos llega a establecer un mapa de mecanismos de crecimiento de grano en el que pueden identificarse tres regiones diferentes (Fig. 1) en función de $R_{\text {med. }}, R_{\text {crít. y }} R_{\text {máx. }}$ La aparición de los distintos tipos de crecimiento de grano es ahora más compleja que la propuesta por Hillert, pues no depende sólo del tamaño de grano crítico sino de la heterogeneidad del sistema.

Aunque ya se apuntó en la descripción de la teoría de Hillert, conviene poner énfasis en que usualmente (y en el caso de aceros microaleados también ocurre) las partículas de segunda fase son inestables, es decir, se disuelven o crecen en función de la temperatura y el tiempo. Ello hace que el tamaño de grano crítico, $R_{\text {crít. }}$ también sea dependiente de ambos factores. Una correcta definición de éste pasa por determinar la evolución de la fracción de precipitados y su tamaño con el tiempo y la temperatura. Si bien existen estudios experimentales de la evolución del tamaño de algún tipo específico de precipitados (en lo que a aceros microaleados se refiere) (10 y 11), son prácticamente inexistentes los relativos a la evolución de la fracción en volumen. Ello se entiende por la complejidad que entraña eliminar del

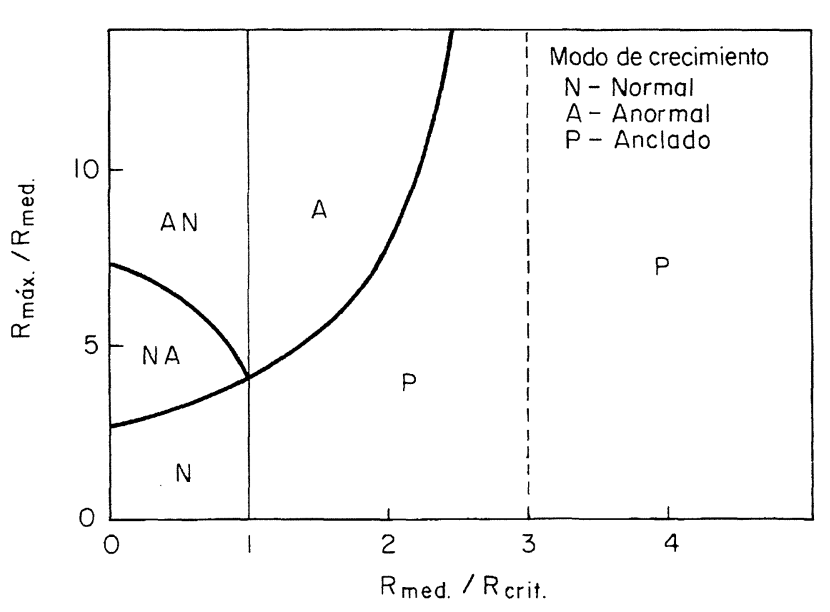

FIG. 1.- Mapa de mecanismos de crecimiento de grano según Ríos (9).

FIG. 1.- Map of grain growth mechanisms after Ríos (9).

cálculo los precipitados no efectivos, es decir, las precipitaciones preferenciales sobre borde de grano y aquellos precipitados que en algunas situaciones se heredan desde la propia solidificación y que son demasiado grandes como para afectar al crecimiento del grano.

También es importante resaltar que $R_{\text {med. }}$ es el tamaño de grano medio de una matriz en la que existen unos granos grandes en crecimiento. Para distribuciones, en las cuales el volumen ocupado por los granos más grandes es pequeño, probablemente no habrá mucha dificultad en medir un $R_{\text {med. }}$ promedio para toda la muestra. Sin embargo, cuando la distribución consiste en unos pocos granos grandes que ocupan una elevada proporción del volumen total, no debe esperarse que el tamaño de grano medio sea representativo del tamaño de grano de la matriz. Esto enlaza directamente con el problema de la medida de los tamaños de grano y con la caracterización del tamaño de grano medio.

\section{LA MEDICIÓN DEL TAMAÑO DE GRANO}

Teóricamente, el tamaño de grano puede expresarse en términos de parámetros lineales, planimétricos o volumétricos. En metalografía es usual representar el tamaño de grano por el diámetro medio del mismo, por su volumen (muchas veces se usa la inversa del volumen, esto es, el número de granos por unidad de volumen, $N_{\mathrm{V}}$ ) o bien por el número equivalente ASTM.

Habitualmente, los tamaños de grano se determinan en 2-D por alguno de los siguientes métodos:

- Contando el número de granos comprendidos en un área dada. 
- Contando la longitud de las intersecciones de rectas trazadas al azar sobre la muestra.

- Contando puntos triples (intersección de tres granos) en un área dada.

- Comparando con patrones estándar.

Pero la hipótesis oculta (12) en estas formas de medir el grano es que hay un tamaño de grano medio que es verdaderamente representativo de la estructura. Como ya se ha avanzado, no siempre los tamaños de grano son uniformes y aún menos en presencia de crecimiento de grano anormal. El uso de estos métodos en tales situaciones puede dar lugar a resultados erróneos.

En general, las medidas en 3-D (que por otra parte son las verdaderamente interesantes) son proporcionales a medidas 2-D ó 1-D y se han desarrollado múltiples expresiones correlativas, aunque todas ellas exigen el conocimiento de un factor de forma.

Sin embargo, como demostraron Núñez y Domingo (13 y 14), no todos los granos de una misma matriz han de tener el mismo factor de forma, de manera que puede determinarse una distribución de factores de forma para una muestra dada. En su análisis, además, dejan claro que los granos no tienen formas geométricas sencillas, sino al contrario. Del mismo modo, tampoco apreciaron ningún tipo de correlación entre los tamaños de los granos y su factor de forma, es decir, todas las formas son posibles a todos los tamaños.

Aceptado, por lo tanto, que un material policristalino homogéneo no tiene por qué presentar una uniformidad de tamaños de grano (aun en ausencia de crecimiento anormal), cabe preguntarse qué tipo de distribución siguen los granos. Son numerosas las observaciones experimentales, en 2-D, que aproximan tal distribución a una de tipo lognormal o similar (15). Dicho de otro modo, el logaritmo de los tamaños de grano se ajusta a una ley normal.

Si bien el problema de medir el grano es en sí independiente de la normalidad o anormalidad del mismo, lo que tiene un verdadero interés práctico es poder dilucidar cuantitativamente cuando un grano es normal o anormal, es decir, distinguir las dos familias de grano cuando éstos son medidos (aunque sea en 2-D). Teóricamente, debe esperarse una distribución de tipo bi-modal (Fig. 2). Sin embargo,

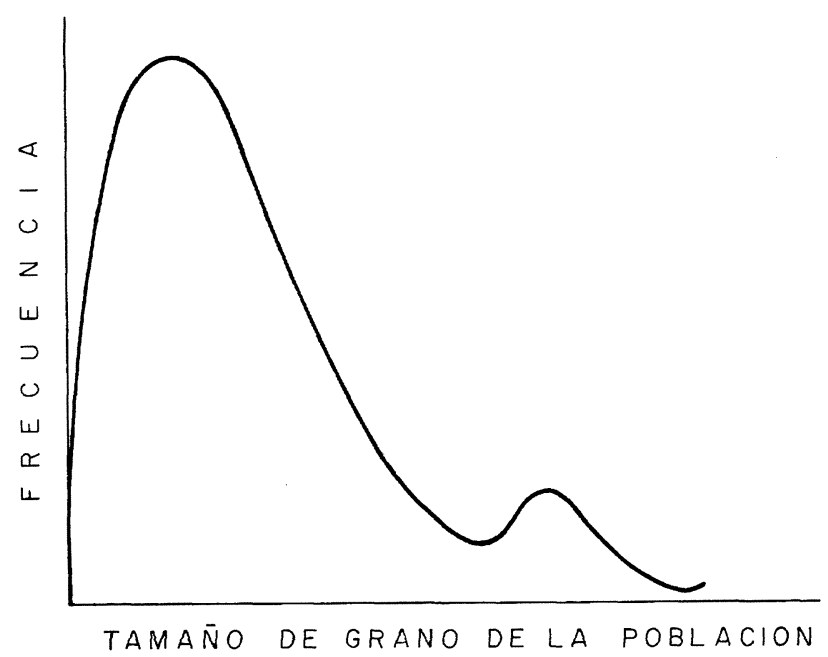

FIG. 2.- Hipotética distribución bimodal del tamaño de grano que cabría esperar en presencia de crecimiento anormal.

FIG. 2.- Theoretical bimodal grain size distribution in the presence of abnormal grain growth

esto es para toda la población, y en la práctica se dispone de una muestra donde los valores de tamaño de grano anormal son poco frecuentes, es decir, que quedan prácticamente integrados en la cola derecha de la distribución global.

Cabe entonces pensar en una forma diferente de representación para poner de manifiesto estos granos anormales. Habitualmente, algunos autores ponderan la frecuencia por el tamaño de grano (16). Sin embargo, esta metodología implica una separación arbitraria de ambas familias, una separación gráfica y visual, sin ningún criterio matemático claro.

Parece claro que para efectuar una caracterización del tamaño de grano anormal se debe proceder a efectuar un estudio detallado de las colas de la distribución de los tamaños de grano de toda la muestra.

\section{MÉTODO EXPERIMENTAL}

Para el presente estudio de crecimiento de grano en aceros microaleados, se seleccionaron dos calidades de un acero microaleado de contenido medio de carbono (38MnSiVS5), cuyas composiciones y denominaciones se presentan en la tabla I.

TABLA I.- Composición química de los aceros objeto de estudio

TABLE I.-Chemical composition of the selected steels

\begin{tabular}{|c|c|c|c|c|c|c|c|c|c|}
\hline Peso, \% & C & Mn & Si & P & S & V & Al & Ti & $\begin{array}{c}\text { N, } \\
\text { ppm }\end{array}$ \\
\hline Acero A & 0,34 & 1,52 & 0,72 & 0,025 & 0,025 & 0,083 & 0,0145 & 0,0181 & 114 \\
Acero B & 0,36 & 1,35 & 0,71 & 0,018 & 0,011 & 0,09 & 0,0224 & 0,0171 & 139 \\
\hline
\end{tabular}


Se debe destacar la presencia de tres elementos microaleantes (vanadio, titanio y aluminio) y los mayores niveles de nitrógeno y aluminio para el acero B. El objetivo de este trabajo es analizar cómo influyen los carbonitruros de V-Ti y los nitruros de aluminio en el tamaño de grano y en la aparición del crecimiento anormal, mediante el estudio de la evolución de las distribuciones de tamaño de grano con el tiempo y con la temperatura.

A partir del material original (redondos laminados de un lingote de $4.000 \mathrm{~kg}$ a $100 \mathrm{~mm}$ de diámetro, con un calentamiento a $1.250{ }^{\circ} \mathrm{C}$ y una temperatura de acabado de $900{ }^{\circ} \mathrm{C}$ ), se extrajeron las probetas de estudio para los tratamientos de solubilización.

Las temperaturas de austenización escogidas fueron las siguientes:

Acero A: $850,900,950,1.000,1.050,1.100$, 1.150 y $1.250^{\circ} \mathrm{C}$.

Acero B: $950,1.000,1.050,1.100,1.150 \mathrm{y}$ $1.250^{\circ} \mathrm{C}$.

Los tiempos de mantenimiento ensayados para cada temperatura fueron $5,10,15,30,60,90$ y 180 min.

Los tratamientos de austenización se efectuaron en un horno tubular y en atmósfera protectora de argón para evitar posibles decarburaciones. Acabada la solubilización, se procedía a un temple directo en agua a temperatura ambiente.

Tras desbaste y pulido, las probetas se sometían a ataque químico con objeto de determinar el tamaño de grano austenítico. Los mejores resultados se obtuvieron mediante ataque en una solución saturada de ácido pícrico (80 c.c.) con adición de jabón líquido (20 c.c.) como agente humectante y ácido clorhídrico (4 gotas de $\mathrm{HCl}$ al $35 \%$ ). El ataque debe efectuarse en caliente $\left(\approx 80^{\circ} \mathrm{C}\right)$ y la superficie de la probeta se debe ir limpiando de forma sistemática, por ejemplo con un pincel, para eliminar la capa pasivante que se produce.

Para la medición del grano se utilizó un analizador automático de imágenes por ordenador. Se determinó el área que ocupaba cada grano y se calculó el diámetro equivalente, con lo que se obtuvieron las correspondientes distribuciones de tamaño de grano (en promedio se midieron 300 granos por condición de ensayo).

\section{RESULTADOS Y DISCUSIÓN}

En la figura 3 se muestra, para varios casos representativos de los ensayados, cómo los histogramas del logaritmo del tamaño de grano se ajustan bien a una ley normal, como se referencian habitualmente. En el presente trabajo se pretenden aprovechar las características de las leyes normales para estudiar las colas de las mismas. Para ello, se tomarán como candidatos a granos anormales aquellos que sean mayores que el valor medio más dos desviaciones estándar de la distribución de logaritmos de tamaños de grano. Si en vez de la media y desviación de la muestra se conocieran las de la población, podría afirmarse con una confianza del 97,7 \% (17) que los granos en tal situación no pertenecen a la población. La hipótesis implícita es que la media y desviación de la población están bien estimadas por las de la muestra. Este criterio de separación, que por otro lado es bastante conservador, permitirá tener una primera evaluación de los granos candidatos a ser anormales.

En la figura 4 se muestra, para algunos de los tiempos ensayados, la evolución con la temperatura del tamaño de grano medio de todos ellos $\left(T G_{\text {normal }}\right)$ y del tamaño medio de la cola derecha de la distribución ( $\left.T G_{\text {anormal }}\right)$ separada según el criterio anterior.

No obstante, estas representaciones gráficas no permiten determinar de forma inequívoca en qué situaciones se está en caso de crecimiento anormal. De esta manera, se ha podido detectar crecimiento anormal en zonas de estas gráficas donde no hay una gran diferencia entre $T G_{\text {normal }}$ y $T G_{\text {anormal }}$, como, por ejemplo, en el acero A austenizado a $950{ }^{\circ} \mathrm{C}$ durante 30 min. Un análisis más apropiado consiste en una representación de las diferencias relativas entre ambas familias de granos, esto es, $\left[\left(T G_{\text {anormal }}-T G_{\text {nor- }}\right.\right.$ $\left.\mathrm{mal}) /\left(T G_{\text {normal }}\right)\right]$. Las diferencias absolutas dependen del propio tamaño de grano, y es por ello que no son útiles.

En la figura 5 se representa, para diversos casos de los estudiados, la evolución de las diferencias relativas con la temperatura. Puede observarse cómo son aproximadamente constantes hasta un valor que coincide con la aparición del crecimiento anormal. En ese momento, las diferencias relativas crecen alcanzando un máximo, después del cual retornan a los valores iniciales y de nuevo se mantienen constantes. Esto último ocurre cuando los granos anormales son de nuevo integrados en la distribución de tamaños de grano. La combinación entre las observaciones experimentales de la microestructura con representaciones gráficas similares a las de la figura 5 , permite afirmar que el crecimiento de grano anormal aparece cuando las diferencias relativas alcanzan un valor superior a 2,5, aproximadamente. Cuando esto ocurre, los tamaños de granos candidatos a ser anormales se tomarán, de hecho, como anormales, mientras que para valores inferiores deben considerarse de la población normal.

Tomando, por lo tanto, este valor de diferencias relativas como criterio de separación del crecimiento normal-anormal, se pueden construir representaciones gráficas de crecimiento de grano anormal- 


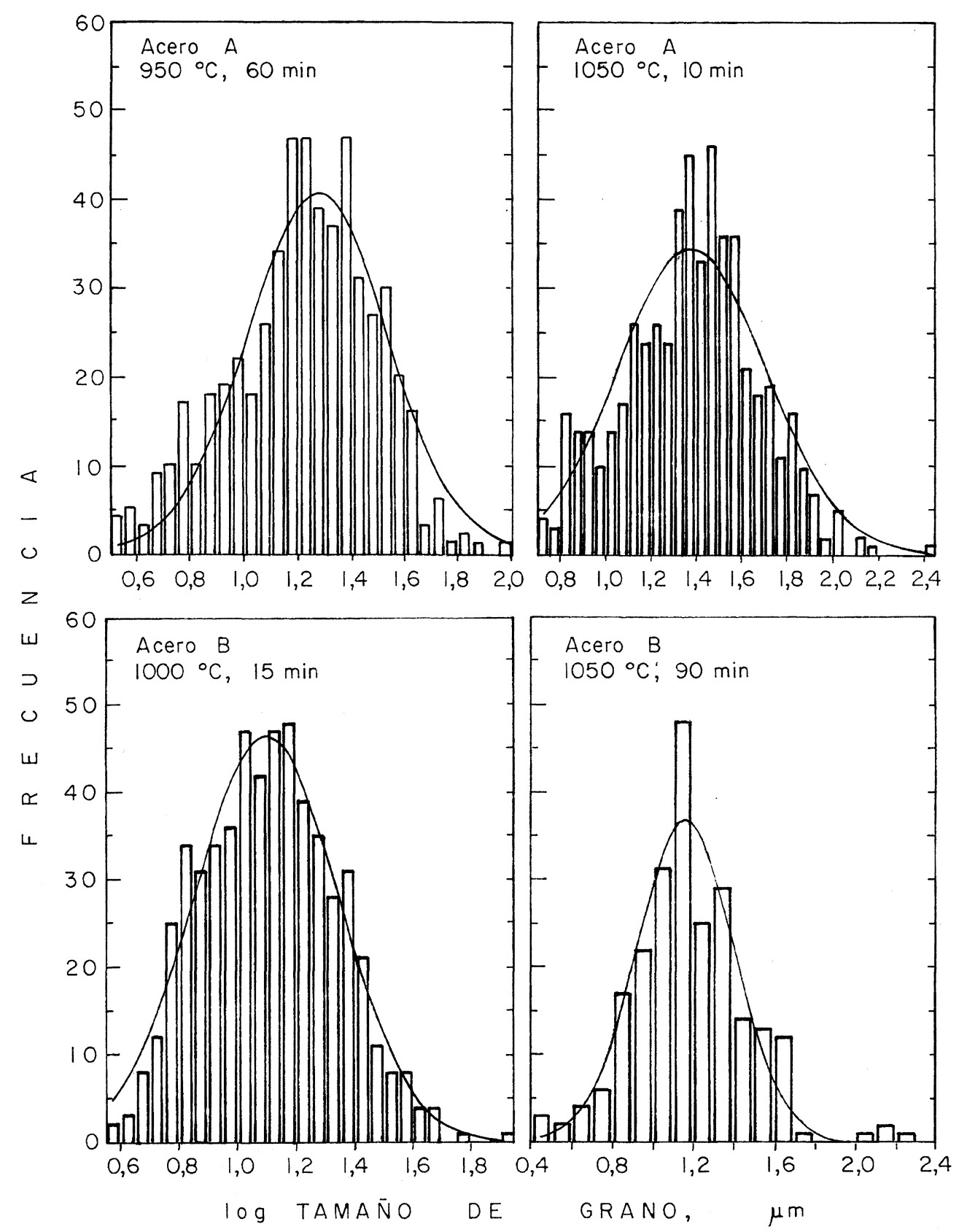

FIG. 3.- Distribución del logaritmo del tamaño de grano (diámetro) para algunas de las situaciones ensayadas.

Fig. 3.- Distribution of the logarithm of grain size (diameter) for some of the cases tested in this work.

tiempo-temperatura (Fig. 6) para cada uno de los aceros en estudio. Debido a que la única diferencia aparente entre ambos aceros es la diferente concentración de nitrógeno y aluminio, cabe atribuir a ello la diferencia de comportamiento observada en la citada figura 6. Para confirmar este punto se procedió a efectuar un estudio adicional teniendo en cuenta las teorías de control de tamaño de grano descritas anteriormente.

Para obtener la fracción de volumen de precipitados presentes a cada temperatura se aplicó el mode- lo de precipitación de Hillert ỳ Staffanson (18-20). El modelo asume una solución regular de un precipitado sencillo de vanadio o titanio, de manera que forma un precipitado complejo de V-Ti. A este modelo se añadió el AlN (ver ecuación [10]). Como productos de solubilidad de los carbonitruros de VTi se tomaron los de la referencia (21), y para el del nitruro de aluminio se tomó el valor usual (22).

$$
\mathrm{V}_{\mathrm{a}} \mathrm{Ti}_{1-\mathrm{a}} \mathrm{C}_{\mathrm{b}} \mathrm{N}_{1-\mathrm{b}}+\mathrm{AlN}
$$






FIG. 4.- Evolución del tamaño de grano (diámetro) con la temperatura. Se compara la media de toda la distribución ( $T G_{\text {normal }}$ ) con la media de la cola derecha de la distribución ( $\left.T G_{\text {anormal }}\right)$.

FIG. 4.-Evolution of grain size (diameter) versus temperature. The mean grain size for the whole distribution $\left(\mathrm{TG}_{\text {normal }}\right)$ is compared with the mean grain size of the right tail of the distribution

$$
\left(\mathrm{TG}_{\text {anormal }}\right)
$$

En la figura 7 se muestran los resultados obtenidos y se observa como ambos aceros presentan los mismos contenidos de precipitados de V-Ti; la única diferencia estriba en la diferente concentración del AlN, tal y como ya se había apuntado. Pero lo que es verdaderamente destacable es la rápida disolución del AlN, al contrario de lo que ocurre con los carbonitruros de V-Ti que presentan una disolución más progresiva. También es de destacar la gran estabilidad de los nitruros de titanio a altas temperaturas y la de los carburos de vanadio a bajas (coeficientes $a$ y $b$ de la ecuación [10]).

La comparación entre las figuras 6 y 7 deja ahora bien claro que la diferencia de comportamiento tiene que deberse a la diferente estabilidad de los precipitados de AlN.

No obstante, la fracción en volumen de precipitados de V-Ti es prácticamente un orden de magnitud superior a la del AlN. Parece, por tanto, que los carbonitruros de V-Ti deben afectar más al control del
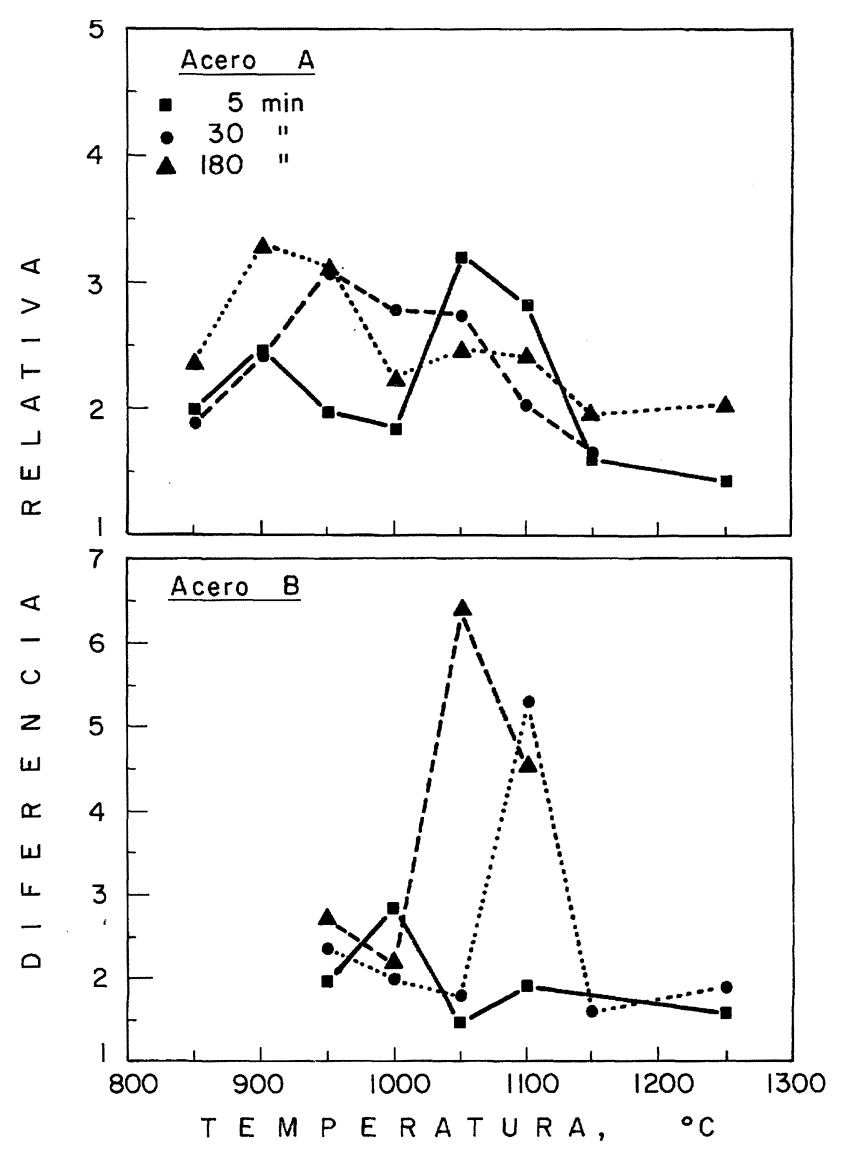

FIG. 5.- Evolución de las diferencias relativas entre los tamaños de grano de toda la muestra y la cola de la misma: [ $\left.\left(T G_{\text {anormal }}-T G_{\text {normal }}\right) /\left(T G_{\text {normal }}\right)\right]$. FIG. 5.-Relative differences between the total grain size and the abnormal candidates: $\left[\mathrm{TG}_{\mathrm{anormal}^{-}}\right.$ $\left.\left.\mathrm{TG}_{\text {normal }}\right) /\left(\mathrm{TG}_{\text {normal }}\right)\right]$

grano que el AlN. Probablemente esto sea así, es decir, el tamaño de grano medio experimental obtenido debe corresponderse con el tamaño de grano crítico determinado por los precipitados de V-Ti. Sin embargo, la repentina disolución del AIN (y por lo tanto el rápido aumento del $R_{\text {crít. }}$ debido exclusivamente a los precipitados de AlN) puede favorecer localmente el crecimiento de algunos granos, promoviendo un aumento de la heterogeneidad del sistema de modo que estos pocos granos que crecen superan la heterogeneidad crítica fijada por los precipitados de V-Ti y así pueden continuar creciendo dando lugar a una microestructura bimodal.

Superponiendo el rango de temperaturas de disolución del AlN sobre los mapas de crecimiento de grano anormal (Fig. 6) pueden diferenciarse tres zonas:

- Temperaturas superiores a las de disolución. La aparición de crecimiento anormal de grano en esta zona donde en principio debe estar totalmente disuelto el AlN, se debe a una situación transitoria. Las probetas se introdujeron directamente 


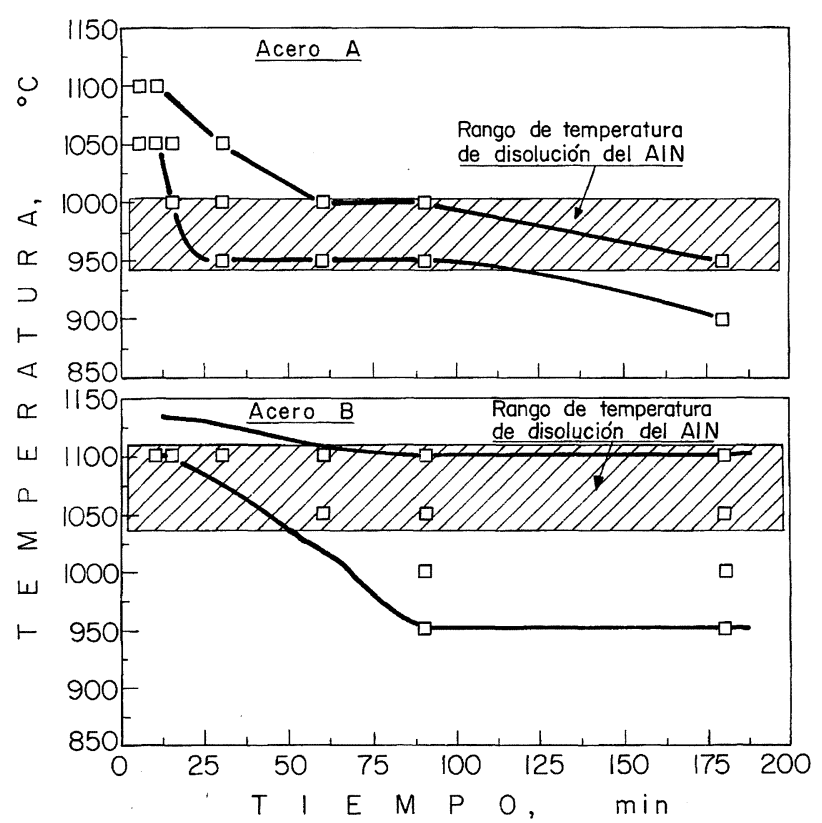

FIG. 6.- Zonas de aparición del crecimiento anormal en función del tiempo y de la temperatura.

FIG. 6.-Zones where the abnormal grain growth is observed in relation with temperature and time.

en el horno a la temperatura de austenización, por lo que es de suponer su sometimiento a elevadas velocidades de calentamiento. Debido a que la disolución requiere de una cinética, el sistema de calentamiento produjo una sobresaturación transitoria de precipitados. El efecto de crecimiento de grano anormal se debe a la disolución del AlN desde este estado de sobresaturación. Con velocidades de calentamiento más lentas este efecto no se habría observado.

- Temperatura de disolución del AlN. Como era de esperar, en esta zona donde desaparece el AlN se produce el crecimiento anormal. Lógicamente, también se observa el estado transitorio, y es por ello que el crecimiento anormal no empieza hasta transcurrido un cierto intervalo de tiempo. Una velocidad de calentamiento menor produciría crecimiento anormal incluso a tiempos de mantenimiento más cortos.

- Temperaturas inferiores a las de disolución. El crecimiento anormal aquí observado no puede deberse a la disolución de precipitados. En este caso, y dado que se observa a tiempos relativamente grandes, es el crecimiento de los precipitados el que afecta al aumento del tamaño de grano crítico, $R_{\text {crít. }}$, debido al AlN.

Para el acero A, la tercera zona se observa sólo ligeramente a tiempos muy largos, debido a la relativamente baja temperatura de disolución de los precipitados. En este caso, la activación térmica necesaria para el crecimiento de los precipitados de AIN no

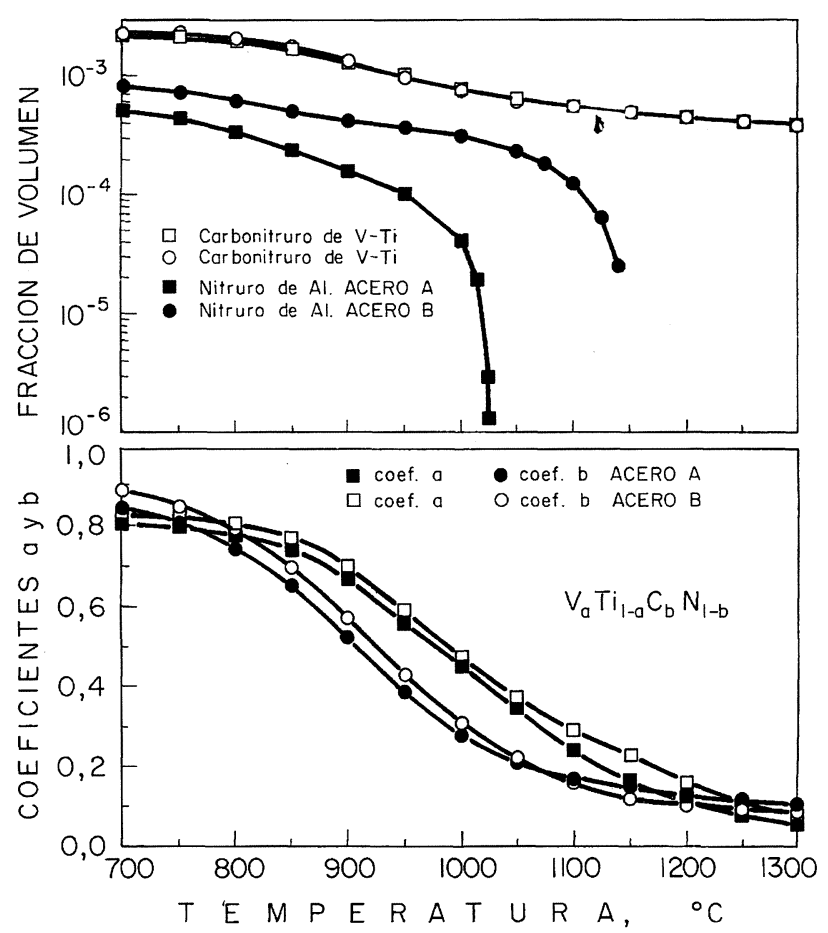

FIG. 7.- Fracción de volumen de los precipitados y de los coeficientes de composición de los mismos en función de la temperatura.

FIG. 7.- Volume fraction of precipitates and their composition coefficients versus temperature.

es suficiente para favorecer su crecimiento. Sin embargo, esta tercera zona es evidente para el acero B. En este caso, los precipitados de AlN se disuelven a mayores temperaturas, $y$, por lo tanto, a temperaturas inferiores todavía existe posibilidad de activación térmica para su crecimiento. La primera zona se observa de forma moderada en el acero B, debido a que el transitorio es más corto a altas temperaturas, y en este caso, además, está muy próxima a la temperatura de disolución. Todo ello queda mucho más claro si se analiza cómo evoluciona el tamaño de grano crítico debido únicamente a los precipitados de AlN. Para ello, se aplicó la expresión del tamaño crítico propuesto por Ríos (ec. [8]). La evolución del tamaño de los precipitados de AlN con el tiempo y con la temperatura se obtuvo de la referencia (11):

$$
\log r=-\frac{5.167}{T}+2.593+\frac{\log t}{3}
$$

donde $r$ es el radio del precipitado (en $\mu \mathrm{m}), T$ es la temperatura (en K) y $t$ el tiempo (en h). Se tomó como fracción de volumen a cada temperatura los valores calculados por el modelo de precipitación de Hillert. En las figuras 8a) y 8b) se comparan, para 

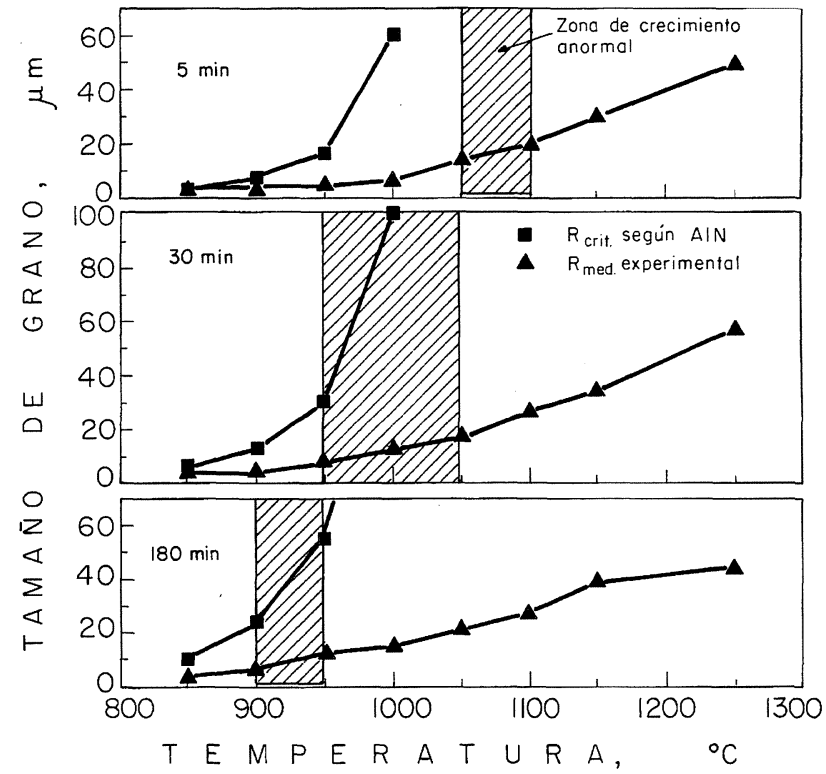

a)
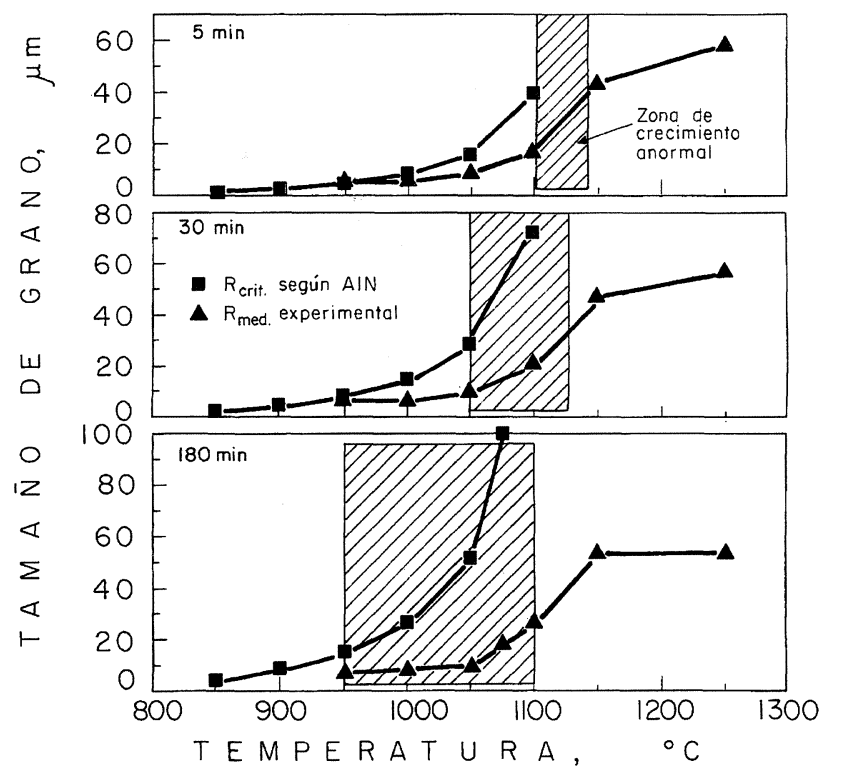

b)

FIG. 8.- Comparación entre el tamaño de grano (radio) experimental y el tamaño de grano crítico determinado por los precipitados de AlN. Puede observarse como el crecimiento anormal aparece cuando el tamaño de grano crítico empieza a crecer, salvo a tiempos cortos debido a la sobresaturación.
a) Acero $A$
b) Acero B.

FIG. 8.- Comparison between the experimental grain size (radii) and the theoretical critical grain size determined by the AlN precipitates. The abnormal growing appears when the critical grain size begins to coarse, except at short times due to the oversaturation.

\section{a) Steel A.}

b) Steel B. algunos tiempos de los estudiados, la evolución del tamaño de grano medido experimentalmente $\left(T G_{\text {nor- }}\right.$ mal $)$ y el tamaño de grano crítico $\left(R_{\text {crít. }}\right)$, debido, exclusivamente, a los precipitados de AlN. Puede apreciarse que para todas aquellas situaciones correspondientes con la zona intermedia anteriormente descrita, el crecimiento anormal coincide con el rápido aumento del tamaño de grano crítico debido al AlN (bien por disolución, bien por crecimiento del precipitado). Cuando predomina la sobresaturación (zona de elevada temperatura y/o tiempos cortos en las otras zonas), la predicción no es correcta, puesto que se minusvalora la cantidad de precipitados presentes. En estas figuras, a largos tiempos (180 min), se observa crecimiento anormal a temperaturas en las que el tamaño de grano crítico determinado por el AIN no ha comenzado a crecer. En estas situaciones no debe descartarse un crecimiento del resto de precipitados de V-Ti. El crecimiento de los mismos también puede provocar un aumento del tamaño de grano crítico debido a estos precipitados complejos de V-Ti y, por lo tanto, favorecer el crecimiento anormal. Este es un hecho que no se ha podido comprobar dada la ausencia en la literatura de expresiones sobre la cinética de crecimiento de este tipo de precipitados.

Es de destacar que una vez superada la zona de crecimiento anormal, el tamaño de grano medio no sufre un crecimiento excesivo, lo que indica que los precipitados complejos de V-Ti todavía tienen un efecto de control. Sin embargo, como demuestra la evolución del tamaño de grano medio experimental con la temperatura para algunos tiempos (Fig. 9), el acero B controla mejor el grano hasta que tiene lugar el crecimiento anormal. A partir de ese momento, el acero A, que ya ha disuelto los precipitados de nitruro de aluminio, ejerce un mayor control. Ello parece indicar que el nitruro de aluminio, aun siendo prácticamente un orden de magnitud menor en fracción de volumen que los carbonitruros de V-Ti, es capaz de efectuar un control efectivo del grano.

Un hecho relevante que cabe comentar es la distinción observada para ambos aceros en los valores de diferencias relativas de las dos familias de granos (Fig. 5). Para el acero B, y especialmente en la zona de disolución teórica de los precipitados, se observan unas diferencias amplias (5-7), mientras que para el acero A, estas diferencias son más bajas (2,5$3,5)$. La razón estriba en la mayor temperatura de aparición del crecimiento anormal en el acero B. Los granos anormales están ahora, además, térmicamente activados para crecer. El sistema propuesto de análisis de las colas de las distribuciones de tamaño de grano ha sido incluso capaz de detectar la aparición de crecimiento anormal moderado, como en el caso del acero A. 


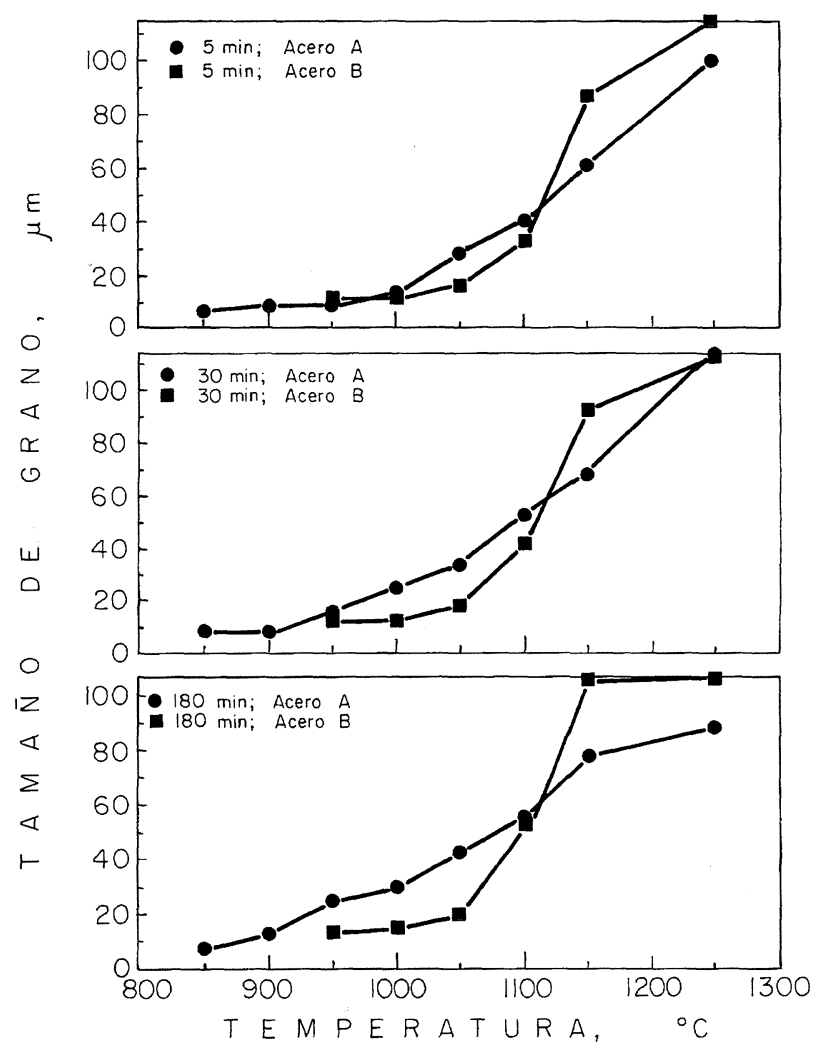

FIG. 9.- Comparación entre ambos aceros del crecimiento de grano (radio) en función de la temperatura.

FIG. 9.- Grain size (radii) of both steels versus temperature.

\section{CONCLUSIONES}

- Para los aceros estudiados, el inicio del crecimiento anormal está gobernado por el $R_{\text {crít. }}$ debido a los precipitados de AlN. A temperaturas cercanas o superiores a las de disolución, el factor gobernante es la fracción en volumen de estos precipitados, mientras que a bajas temperaturas y largos tiempos gobierna el crecimiento de los precipitados, tanto de los de AlN como de los complejos de VTi. La expresión propuesta por Ríos para el $R_{\text {crít. }}$ concuerda con los resultados obtenidos.

- La adición de AlN a los aceros microaleados debe efectuarse con precaución. Las elevadas concentraciones ayudan a controlar el crecimiento de grano, pero trasladan la aparición del crecimiento anormal a altas temperaturas, favoreciendo, por lo tanto, granos extremadamente grandes. Las bajas concentraciones son menos efectivas en el control del grano, pero trasladan el crecimiento anormal a más bajas temperaturas, por lo que el contenido de granos anormales es más moderado que en la situación anterior.

- El sistema propuesto de separación de las colas de la distribución de tamaños de grano puede ser una buena herramienta de análisis si se acompaña de las diferencias relativas entre la media de todos los granos y la media de la cola de la distribución.

- Los mapas de crecimiento anormal-tiempo-temperatura pueden ser un instrumento útil de cara al diseño de las aleaciones y a una mejor comprensión del papel que desempeñan los microaleantes.

\section{Agradecimiento}

Uno de los autores, J.M.C., desea agradecer la concesión de una beca de formación de investigadores por parte de la Direcció General de Recerca de la Generalitat de Catalunya.

\section{REFERENCIAS}

(1) NAYLOR, D.J. Ironmaking Steelmaking, 16 (4), 1989: 246252.

(2) Platts, K, Vassiliou, A.D. y Pickering, F.B. Metall. Mater. Technol., sept., 1984: 447-454.

(3) Korchinsky, M. Proc. 3rd Int. Mini Mills Conference, Nueva Orleáns, mar. 1984.

(4) CotTeRILl, P., y Mould, P.R. Recrystallization and grain growth in metals. Ed. Surrey University Press, Londres, 1976: 266-325.

(5) ZENER, C. Trans AIME, 175, 1948: 47.

(6) HillerT, M. Acta Metall., 13, mar., 1965: 227-238.

(7) Lifshitz, I.M. y Slyozov, V.V. J. Phys. Chem. Solids, 19, 1961: 35

(8) Gladman, T. Proc. R. Soc., 294, 1966: 298-309.

(9) Ríos, P.R. Acta Metall., 35 (12), 1987: 2.805-2.814.

(10) Coladas, R., Masounave, J., Guerin, G. y Bailon, P. Met. Sci., 1977: 509.

(11) Gladman, T. y Pickering, F.B. J. Iron Steel Inst., 205, 1967: 653-664.

(12) Russ, J.C. Practical Stereology. Ed. Plenum Press, Nueva York, 1986: 53-72.

(13) NÚÑEZ, C. y Domingo, S. Metall. Trans., 19A, abr. 1988: 933-940.

(14) NúÑEZ, C. y Domingo, S. Metall. Trans., 19A, dic. 1988: 2.937-2.944.

(15) RaLPH, B. Mater. Sci. Technol., 6, nov. 1990: 1.139-1.144.

(16) KASPAR, R. y MAHMOUD, N. Mater. Sci. Technol., 7, mar. 1991: 249-254.

(17) Box, G.E., Hunter, W.G. y Hunter, J.S. Statistics for Experimenters. Ed. John Wiley \& Sons, Nueva York, 1978: 15-53.

(18) Hillert, M. y Staffanson, L.I. Acta Chem. Scand., 24, 1970: 3.618 .

(19) Adrian, H. Mater. Sci. Technol, 18, may. 1992: 406-420.

(20) Ríos, P.R. Mater. Sci. Technol., 4, abr. 1988: 324-327.

(21) Adrian, H. y PiCKering, F.B. Mater. Sci. Technol., 17, feb. 1991: 176-182.

(22) Wilson, F.G. y Gladman, T., Int. Mater. Rev., 33 (5), 1988: 221-286. 